\title{
Materials Defects and Society
}

Shortly after I started to learn about crystal defects, it occurred to me that materials scientists have at their fingertips a readymade analogue of society. At the simplest level, a perfect crystal-viewed for the moment as a continuum with particular properties-is rather like the society we live in, with its particular cultural attitudes. At room temperature, it is thermodynamically inevitable that there will be point defects-or individuals, in my model. The behavior of these individuals is constrained by society; that is, they belong to a specific crystal. However, there are many types of defect, which demonstrate many different behaviors.

Let's take the simplest case of a monatomic crystal-for instance, a metal rather than an alloy. This will have single vacancies, which will in some cases meet others and bind together to form di-vacancies
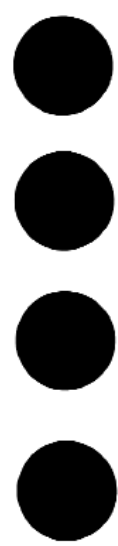
(couples, as we might call them). Tri-vacancies exist (as do ménages à trois), but they are rare. Then we have impurity atoms or deliberate dopants, which can occupy interstitial or substitutional sites. We might call these heteroatoms. Interstitial hetero-atoms could be those from another culture (non-scientists perhaps) who have not succeeded in adopting the behaviors of the host culture (although they could, as in real materials life, be extremely useful). Substitutional atoms have adopted the cultural values of the host society but are still detectably different in some respects.

Of course, hetero-atoms may find it thermodynamically favorable to congregate, such as in clubs, quarters (such as "the Latin quarter"), or eventually in independence movements. These are our segregates, precipitates, or eventually large regions of a new phase.

In real crystals, temperature plays a big role in determining behavior, or at least controlling the rate at which things happen. It is the same in society, if we consider temperature as per-capita disposable income. In richer societies (hotter crystals), mobility is higher, and so (incidentally) is the divorce rate (or di-vacancy dissociation rate). I am not sure whether indepen-
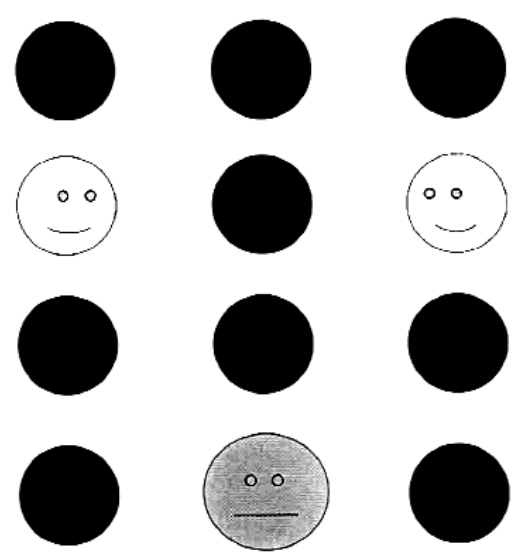

dence movements develop faster in rich countries, but no analogy is perfect!

How might this analysis apply to materials science itself? We certainly have our own crystal structure (set of values) and it certainly contains its heteroatoms (those who question those values and seek to change them). In my own parochial areas (electron microscopy and undergraduate education), it seems that we are stronger for the presence of the hetero-atoms. As materials scientists we would, of course, expect this.

The role of change agents such as lobby groups and special interest groups (SIGs) can also be explained-they are dislocations. These are thermodynamically unlikely defects which have been introduced (deliberately in this case) in order to effect change. They do this by moving through the crystal (society), slightly modifying the positions of a small percentage of the point defects (individuals) at any one time but, over time, touching a majority of people. The direction of the dislocation, and its Burgers vector, are influenced (if not completely controlled) by external factors. Just as a crystal dislocation moves in response to the application of an external stress, so SIGs move in directions encouraged by government policy, society's evolving attitudes, and even debate at Materials Research Society meetings. It is to be hoped that the directions of these external forces are not reversed too often or those of us at the core of the dislocation may get confused (or even fatigued)! Unlike atoms and vacancies, we tend to have our own beliefs.

Of course, any movement or SIG which would like eventually to see substantial changes should consider themselves not as a single dislocation, but as a source of dislocations. I am unsure, yet, how the source works, but the societal analogue of a Frank-Read source might be described as "what goes around comes around."

My crystal analogy can be extended in several ways, which I leave to the rich imaginations of my readers. Alloys? Ordered crystals? Twins? Grain boundaries? Phase boundaries? Ostwald ripening? Orowan loops (when a lobby group tries to surround us)? Voids? Recrystallization? I am a bit frightened of melting and re-solidification because I am not at all sure that the new crystalline material would be any better to be a vacancy in than the existing one.

Now I am going to leave the keyboard so I can interact with the other half of my di-vacancy.

Peter GOODHEW 